\title{
Modelling and observations: Comparison of the magnetic field properties in a prominence
}

\author{
D. H. Mackay ${ }^{1}$, B. Schmieder ${ }^{2}$, A. López Ariste ${ }^{3}$, and Y. $\mathrm{Su}^{4,5}$ \\ ${ }^{1}$ School of Mathematics and Statistics, University of St Andrews, North Haugh, St Andrews, Fife KY16 9SS, UK \\ e-mail: dhm@st-and.ac.uk \\ 2 LESIA, Observatoire de Paris, PSL Research University, CNRS, Sorbonne Universités, UPMC Univ. Paris 06, Univ. Paris Diderot, \\ Sorbonne Paris Cité, 5 place Jules Janssen, 92195 Meudon, France \\ e-mail: brigitte.schmieder@obspm.fr \\ 3 IRAP, Université de Toulouse, CNRS, CNES, UPS, 14 Av. E. Belin, 31400 Toulouse, France \\ e-mail: Arturo.LopezAriste@irap.omp.eu \\ ${ }^{4}$ Key Laboratory of Dark Matter and Space Astronomy, Purple Mountain Observatory, Chinese Academy of Sciences, \\ Nanjing 210008, PR China \\ 5 School of Astronomy and Space Science, University of Science and Technology of China, Hefei, Anhui 230026, PR China
}

Received 9 September 2019 / Accepted 16 March 2020

\begin{abstract}
Context. Direct magnetic field measurements in solar prominences occur infrequently and are difficult to make and interpret. As a consequence, alternative methods are needed to derive the main properties of the magnetic field that supports the prominence mass. This is important for our understanding of solar prominences, but also for understanding how eruptive prominences may affect space weather.

Aims. We present the first direct comparison of the magnetic field strength derived from spectro-polarimetric observations of a solar prominence, with corresponding results from a theoretical flux rope model constructed from on-disc normal component magnetograms.

Methods. We first used spectro-polarimetric observations of a prominence obtained with the magnetograph THEMIS operating in the Canary Islands to derive the magnetic field of the observed prominence by inverting the Stokes parameters measured in the He D3 line. Next, we constructed two data-constrained non-linear force-free field (NLFFF) models of the same prominence. In one model we assumed a strongly twisted flux rope solution, and in the other a weakly twisted flux rope solution.

Results. The physical extent of the prominence at the limb (height and length) is best reproduced with the strongly twisted flux rope solution. The line-of-sight average of the magnetic field for the strongly twisted solution results in a magnetic field that has a magnitude of within a factor of 1-2 of the observed magnetic field strength. For the peak field strength along the line of sight, an agreement to within $20 \%$ of the observations is obtained for the strongly twisted solution. The weakly twisted solution produces significantly lower magnetic field strengths and gives a poor agreement with the observations.

Conclusions. The results of this first comparison are promising. We found that the flux rope insertion method of producing a NLFFF is able to deduce the overall properties of the magnetic field in an observed prominence.
\end{abstract}

Key words. Sun: filaments, prominences - Sun: magnetic fields

\section{Introduction}

Solar prominences (as observed on the limb) or filaments (on the disc) are cool dense plasma structures that exhibit a wide variety of morphologies (Tandberg-Hanssen 1995; Labrosse et al. 2010; Engvold 2015). The importance of magnetic fields for the existence of solar prominences or filaments was first noted from the on-disc photospheric magnetic field observations of Babcock \& Babcock (1955). These observations showed that solar filaments always formed above polarity inversion lines (PILs) of the normal magnetic field component at the photosphere. This means that filaments formed at locations where the coronal magnetic field was inferred to be mainly horizontal. It is now known that magnetic fields are fundamental to the existence and structure of solar prominences. They support the dense prominence plasma against gravity and also insulate it from the surrounding hotter $1 \mathrm{MK}$ coronal plasma. Following the observations of Babcock \& Babcock (1955), an idealised magnetic field model of solar prominences was developed by Kippenhahn \& Schlüter (1957; see also Kuperus \& Raadu 1974).

The first direct measurements of the magnetic field in prominences using the Hanle effect occurred in the 1970s at the Pic du Midi observatory (Leroy 1977; Sahal-Brechot et al. 1977). These and subsequent studies showed that (i) quiescent prominences have a field strength of $3-15 \mathrm{G}$, (ii) the field is mostly horizontal, (iii) the horizontal field makes an acute angle of about $36^{\circ}$ to the long axis of the prominence (Bommier et al. 1994; Bommier \& Leroy 1998), (iv) within the prominence, the field strength increases slightly with height, and (v) the majority of prominences have inverse polarity. Full details of these studies along with further references can be found in the reviews of Leroy (1989) and Paletou \& Aulanier (2003). In recent years these early observations have been verified through a number of studies using the $\mathrm{He}_{3}$ line (Casini et al. 2003; López Ariste \& Casini 2002, 2003; López Ariste \& Aulanier 2007) along 
with the production of full vector magnetic field maps within prominences. Since 2012, the French telescope, Télescope Héliographique pour l'Etude du Magnétisme et des Instabilités Solaires (THEMIS), in the Canary Islands has observed prominences during international campaigns using the MTR (multi raies) mode. More than 200 prominences have been observed in $\mathrm{He} \mathrm{D}_{3}$, from which statistical results have been presented during the IAU S300 symposium (Lopez Ariste 2014; Schmieder et al. 2015), and case studies have been published (Schmieder et al. 2013, 2014; Levens et al. 2016a,b). These maps have shown that the strength of the magnetic field in prominences can vary significantly over short distances. This is challenging to explain for theoretical models.

The observations above provided the impetus for a wide range of theoretical models for explaining the magnetic structure of filaments and prominences. A full review of these models can be found in Tandberg-Hanssen (1995) and Mackay et al. (2010). Early modelling attempts mainly considered idealised magnetic field configurations, but in recent years, a wide range of data-constrained and data-driven models have been developed. In these data-constrained models, observed normal component magnetograms are applied as a photospheric boundary condition from which a static coronal magnetic field is extrapolated. The extrapolated field is normally made using the linear forcefree (LFF), linear magnetohydrostatic (LMHS), or the non-linear force-free (NLFF) field approximation. One of the most successful static extrapolation models is that of Aulanier \& Démoulin (1998; see also Aulanier \& Démoulin 2003; Dudík et al. 2008). It has reproduced many of the observed features of individual solar prominences, such as the feet or barbs, along with breaks in the filament body. This has mainly been carried out through considering the location and distribution of dips in the magnetic field lines (see also Mackay \& van Ballegooijen 2009 for a nonstatic model).

van Ballegooijen (2004) put forward an alternative method for the construction of NLFF fields called the flux rope insertion model. This model is again data constrained where an observed photospheric magnetogram is used to construct an initial potential field. To reproduce the non-potential structure of a filament, a magnetic flux rope with specified axial and poloidal flux is inserted. Following the insertion, the coronal field is relaxed to an equilibrium state using magneto-frictional relaxation, during which the photospheric boundary is held fixed. Within this approach the amount of axial and poloidal flux may be varied until a good fit with observations is found. This method was successfully applied to a U-shaped filament with a barb, observed by the Swedish Solar Telescope (SST). Further applications of this technique have been used to study filament eruptions and flare ribbons (Su et al. 2009, 2018). Luna et al. (2017) showed that the magnetic field strength and the radius of curvature of an observed filament, deduced from the flux rope insertion method, fitted the same quantities obtained by seismology techniques applied to the filament.

One common feature of the above models is that the comparison between the models and the observed filaments is carried out through plotting dips in magnetic field lines to approximately 1 pressure scale height to deduce the portion of the field that may contain cool plasma. More recently, a new technique for the visualisation of cool plasma in $\mathrm{H} \alpha$ in magnetic dips, using radiative transfer techniques applied to magnetic dips filled with plasma, has been developed and applied by Gunár \& Mackay (2015a,b, 2016). Gunár et al. (2018) successfully compared this visualisation technique along with field lines deduced from a magnetohydrodynamic extrapolation model to an observed filament.
With the data-constrained magnetic field models described above, it is now possible to attempt a direct comparison between the magnetic field distribution in an observed prominence with that of a theoretical model. Therefore we here carry out the first direct comparison between dedicated magnetic field observations within a prominence with the properties of the magnetic field deduced from a novel data-constrained theoretical model. Through this comparison, we aim to obtain a greater insight into the nature of the magnetic fields in solar prominences and stimulate a new generation of theoretical models. At first glance, this comparison should be straightforward. The main difficulty in the comparison is that magnetic field observations of prominences can only be carried out at the limb, while the photospheric magnetic fields required for theoretical modelling can only be made on the disc close to central meridian. Thus the difference between the photospheric data used in the model and the underlying magnetic field of the prominence at the limb taken up to 7 days later may be significant. To minimise these differences, the data-constrained NLFFF modelling technique of Su \& van Ballegooijen (2012) and Su et al. (2015) is applied. This technique has produced highly realistic representations of mid- to high-latitude prominences. After the field is constructed, the configuration is rotated to the limb and compared with the observations. We here carry out a basic comparison as a proofof-concept study.

The paper is structured as follows. Section 2 describes the evolution and properties of the prominence under consideration during its disc-to-limb transition between September 20-24, 2013. Along with this spectro-polametric observations of the prominence taken by THEMIS/MTR and histograms of the distribution of the field strength and inclination of the prominences magnetic field are discussed. Section 3 describes the technique applied to construct the NLFF magnetic field configurations that represent the prominence. Two configurations are considered, one with strong poloidal flux and another with weak poloidal flux. We carry out a detailed analysis of these configurations. In Sect. 4 both a qualitative and quantitative comparison between the observations and model are carried out. Finally, the discussion and conclusions are given in Sect. 5 along with suggestions for possible future studies.

\section{Overview of the prominence observations}

Using multi-instrument observations from both ground and space, Schmieder et al. (2014) studied in detail the dynamics and magnetic field of a prominence that was observed on the limb on September 24, 2013. These observations compared the fine-scale structures of a prominence as seen across multiple wavelengths and showed that parts of the prominence exhibited highly disorganised motions that the authors related to magnetohydrodynamic (MHD) turbulence. In addition to studying plasma motions, Schmieder et al. (2014) also considered the nature of the magnetic field of the prominence as deduced from THEMIS/MTR observations. The results from Schmieder et al. (2014) are discussed below, where the THEMIS/MTR observations relevant to the present study are described in detail.

\subsection{Transition of filament to prominence}

Figure 1 shows a time sequence of $\mathrm{H} \alpha$ images provided by Climso at Pic du Midi of the transition of a filament on the solar disc to a prominence above the limb taken over a 5 day period between September 20-24, 2013. On September 20, 2013, a large inverse U-shaped filament lies between latitudes of 
$20 / 09 / 2013$

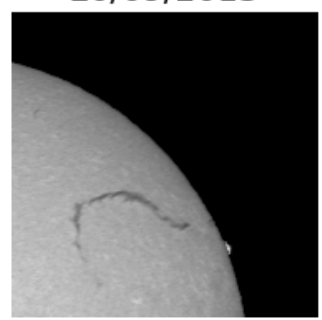

$21 / 09 / 2013$

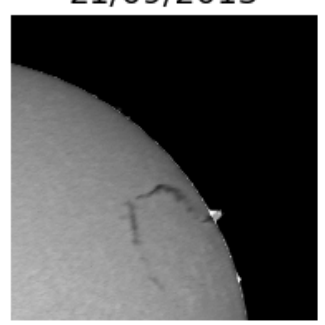

$22 / 09 / 2013$

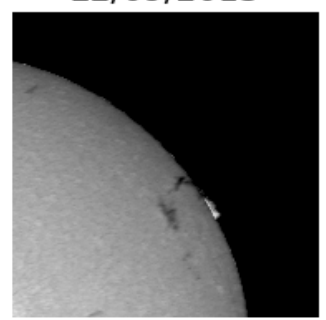

$23 / 09 / 2013$

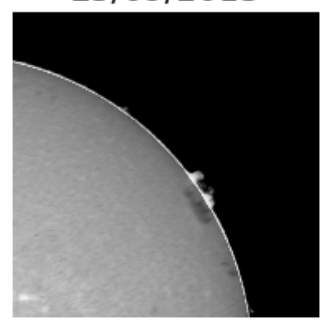

$24 / 09 / 2013$

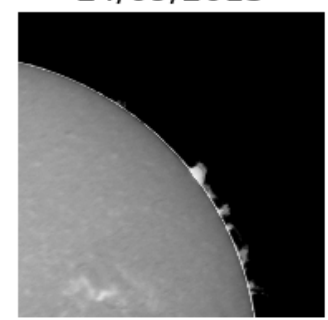

Fig. 1. Evolution of the dark filament viewed in $\mathrm{H} \alpha$ absorption observed on September 20, 2013, by Climso at Pic du Midi. It progressively develops into a bright prominence viewed in $\mathrm{H} \alpha$ emission between September 21-24, 2013.

N20-38 degrees and extends over $35^{\circ}$ in longitude (W35-70) according to the Meudon spectroheliograms ${ }^{1}$. The northern section is mainly extended east-west, where the part of the filament that is closest to central meridian extends from north to south. The filament shape lies along a switchback of the underlying PIL. Over the next four days, the rotation of the Sun gradually transitioned the filament into a prominence on the limb, where the prominence appears approximately between a position angle $(\mathrm{PA})=290-308^{\circ}$ by September 24, 2013.

Figure 2 shows the prominence on September 24, 2013, at 12:22 UT as seen by the Atmospheric Imaging Assembly (AIA; Lemen et al. 2012 on board the Solar Dynamic Observatory (SDO)) in (a) $193 \AA$ where the arcade visibility has been enhanced, (b) $304 \AA$, and (c) a composite 193 and $304 \AA$ image. In each panel the prominence, the prominence cavity, and the corona loops are identified as appropriate for each wavelength. The white box in panel (b), which is orientated parallel to the local limb, denotes the much smaller field of view of the THEMIS/MTR observations (see Sect. 2.2 and Fig. 3). Figure 2a shows that a series of almost concentric coronal loops lies above the prominence at the limb, where the prominence is seen as the dark absorption structure. Previous studies (Schmieder et al. 2004) have shown that the dark area in $193 \AA$ (or $171 \AA$ ) closely corresponds to the shape of the prominence observed in $\mathrm{H} \alpha$ (see Fig. 2 or 3 of Schmieder et al. 2014 for the prominence used in this study). The reason for this agreement is that the coronal radiation in $193 \AA$ (and $171 \AA$ ) is absorbed by the cool prominence plasma by photoionisation, where by chance the optical thickness in both of these wavelengths is equal to the optical thickness of $\mathrm{H} \alpha$ (Anzer $\&$ Heinzel 2005). In contrast, the bright prominence in $304 \AA$ and $\mathrm{He}_{3}$ is produced by emission of $\mathrm{He}$ as a result of incident radiation. Correspondingly, the shapes of the prominence in $304 \AA$ and $\mathrm{He}_{3}$ are similar to one another, but both are different from the $\mathrm{H} \alpha$ prominence (Schmieder et al. 2014).

\subsection{THEMIS/MTR observations}

Figure 3 shows THEMIS/MTR observations of the prominence taken between 12:26 and 14:31 UT on September 24, 2013 in the $\mathrm{He} \mathrm{D}_{3}$ line. Figure $3 \mathrm{a}$ shows the intensity of the $\mathrm{He} \mathrm{D}_{3}$ line, where the northern and southern end of the observations are denoted by "N" and "S", respectively. The strongest intensity in $\mathrm{He}_{3}$ is found at the northern end, where the limb view extends down the axis of the prominence, which is oriented from east to west. The THEMIS/MTR He $\mathrm{D}_{3}$ observations include the Stokes parameters from which the strength, inclination, and azimuth of

\footnotetext{
1 http://bass2000.obspm.fr/home.php
}

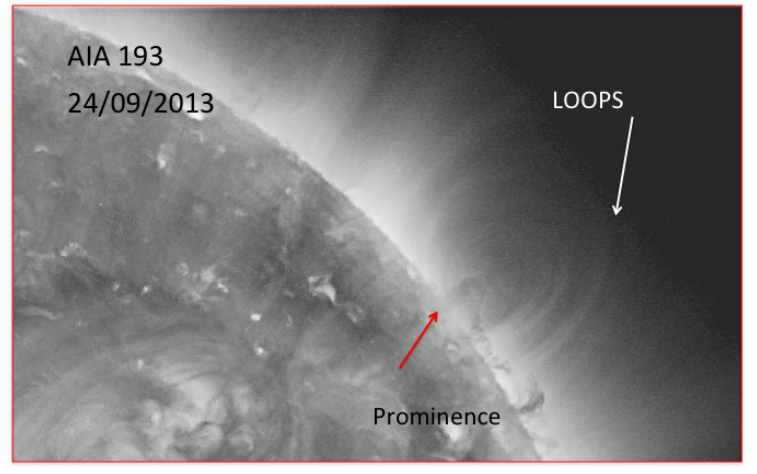

(a)

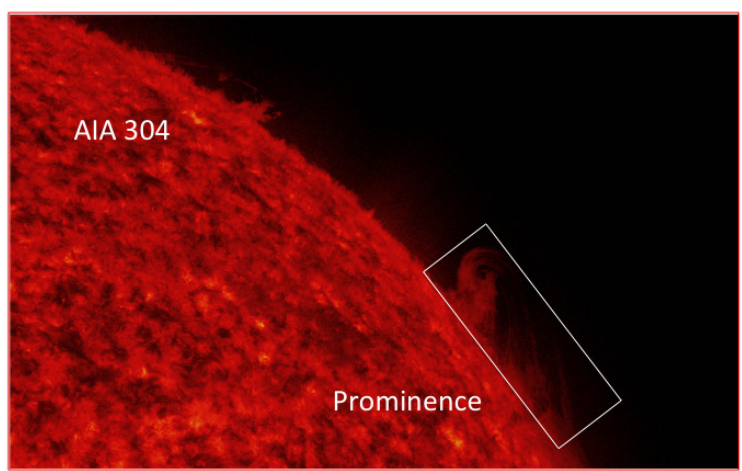

(b)

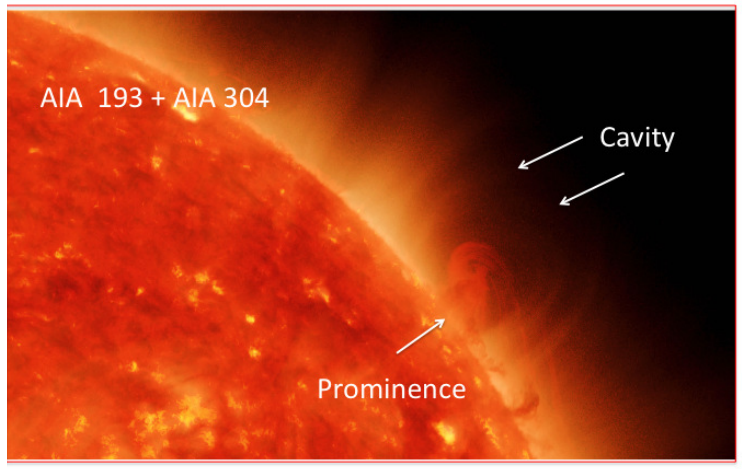

(c)

Fig. 2. Prominence observed on September 24, 2013 at 12:22 UT with AIA in (a) $193 \AA$, where the arcade visibility has been enhanced, $(b)$ $304 \AA$, and $(c)$ a composite 193 and $304 \AA$ image. Panel c: relationship between the prominence, its cavity, and the overlying arcades. The white box in panel $b$ denotes the THEMIS/MTR field of view for observing in the $\mathrm{He}_{3}$ line (see Fig. 3). 


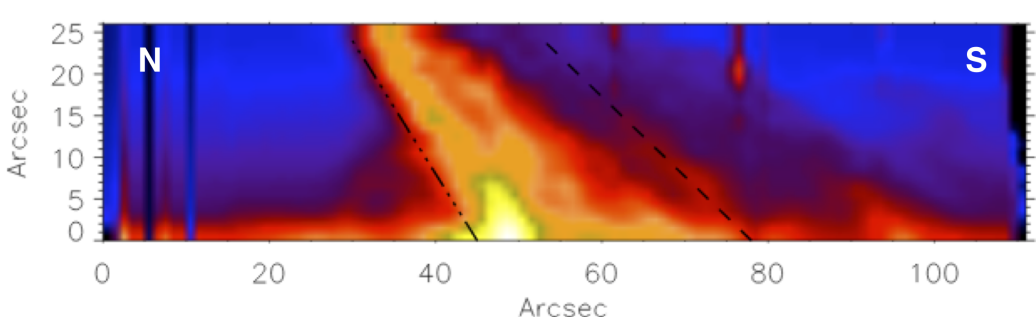

(a)

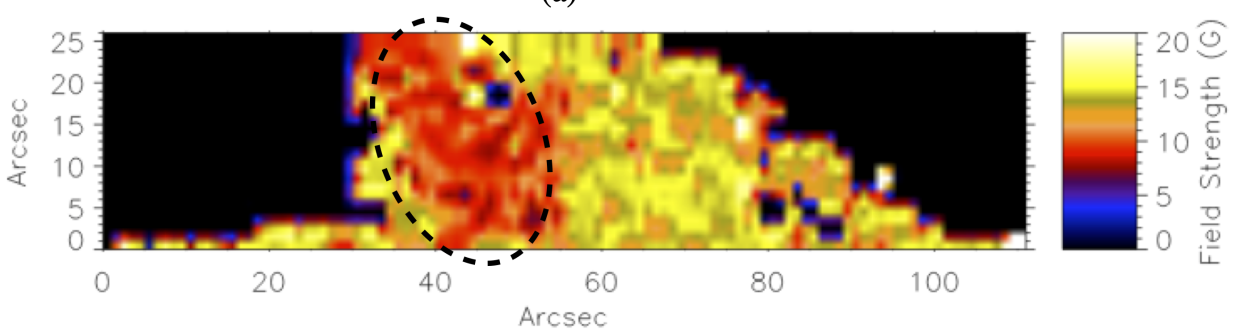

(b)

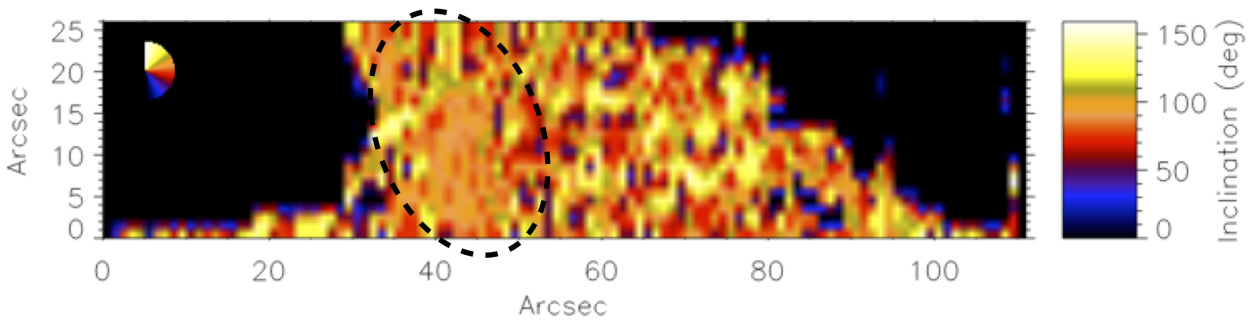

(c)
Fig. 3. Prominence observed on September 24, 2013, by THEMIS/MTR showing (a) the intensity of $\mathrm{He} \mathrm{D} 3$ line, $(b)$ the magnetic field strength in Gauss $(\mathrm{G})$, and (c) the inclination angle of the field to the local vertical. The northern and southern end of the THEMIS/MTR field of view is indicated by "N" and "S" in panel $a$. the prominence magnetic field can be determined (Schmieder et al. 2014). To determine these values, the Stokes profiles are fed into an inversion code based on a principal component analysis (PCA; López Ariste \& Casini 2003; Casini et al. 2003). This compares the observed profile with a database of theoretical profiles produced from the polarised radiative transfer equations of $\mathrm{He} \mathrm{D}_{3}$. The best-fit profile from the database, containing over 90000 profiles, is kept as the solution, while the parameters used to construct the best-fit profile give the inferred vector magnetic field. Error bars are determined for these parameters, as is a statistical analysis on all other model profiles which are sufficiently similar but not as accurate as the best-fit profile. Full details of the MTR data reduction for the prominence in Fig. 3 are given in Schmieder et al. (2013).

We are here mostly interested in the properties of the magnetic field within the prominence, where the magnitude of the magnetic field is given in Fig. 3b. This panel shows that the field strength across the prominence, after line-of-sight integration, varies significantly; most values lie in the range $5-15 \mathrm{G}$. Interestingly, across the prominence two distinct regions can be identified in which the magnetic field strength varies. In the first region, denoted by the dashed oval, the field strength mainly lies in the range $8-11 \mathrm{G}$ and the spatial variation is relatively small. In contrast, outside of the oval the spatial variation of the field strength is much stronger: higher values in the range of $11-18 \mathrm{G}$ occur. Figure $3 \mathrm{c}$ shows the inclination angle of the field, where the inclination angle is defined to be the angle of the magnetic field to the local vertical. Because this prominence lies near to or at the limb, an inclination angle of $90^{\circ}$ indicates that the field is horizontal. Two distinct regions of the prominence can again be identified. The area covered by the oval includes an extended region around the centre of the oval, where the inclination angle is relatively uniform and around $90^{\circ}$, indicating horizontal fields. While this is the case for the majority of the oval, nearer to the upper parts, a stronger spatial variation of the inclination angle is found. The inclination angle outside of the oval also varies strongly spatially, where values typically in the range $40-150^{\circ}$ are found. We also note that panels $b$ and $c$ of Fig. 3 show non-zero values at the top of the THEMIS/MTR field of view between the ranges of 30-70", while outside this range, zero values are obtained. One possible reason for this is that within this range, the prominence extends beyond the THEMIS/MTR field of view. We discuss this further in Sect. 3.

More quantitative information on the properties of the lineof-sight integrated magnetic field in the prominence is shown in Fig. 4, which provides histograms of (a) the field strength and (b) the inclination of the field. In these plots, the red curve and blue histograms are individually normalised such that the integral over each data set is 1 . To fully understand the nature of the magnetic field of the prominence, care must be taken in interpreting the data given by the blue histograms and red curve. The blue histograms represent locations where the error (determined as the variance among the models in the database that best fit the observed profiles, where the best fit is kept as the solution) is smaller than $10 \%$. For these points the errors are consistent with instrumental noise and the finite size of the database used in the fitting. All points for this sample have a high confidence level for the measurement of the magnetic field and thus may be regarded as reliable measurements. For these measurements, most values of the field strength (Fig. 4a) are in the range of approximately $12-15 \mathrm{G}$. However, measurements can lie anywhere in the range $8-19 \mathrm{G}$. The distribution also has two clear gaps where no values are present, below $8 \mathrm{G}$ and between $9-11.5 \mathrm{G}$. The reason for the absence of any measurements within the latter band is unclear. 


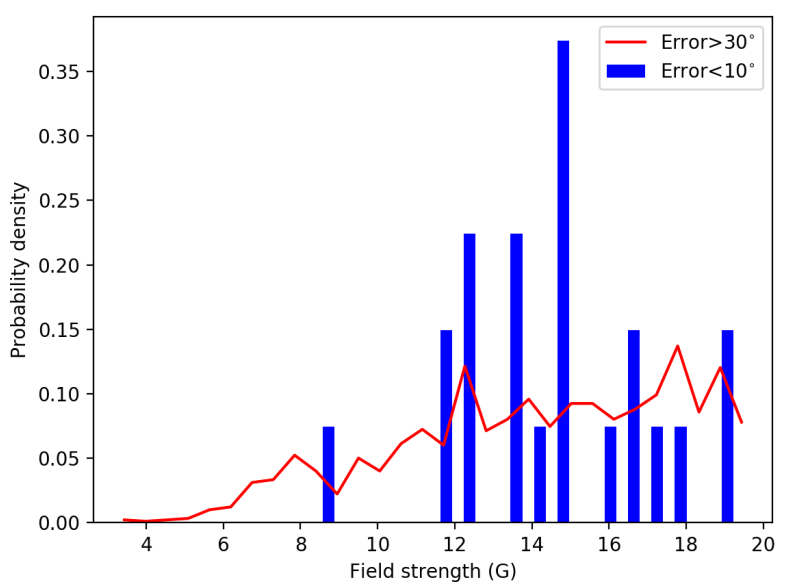

(a)

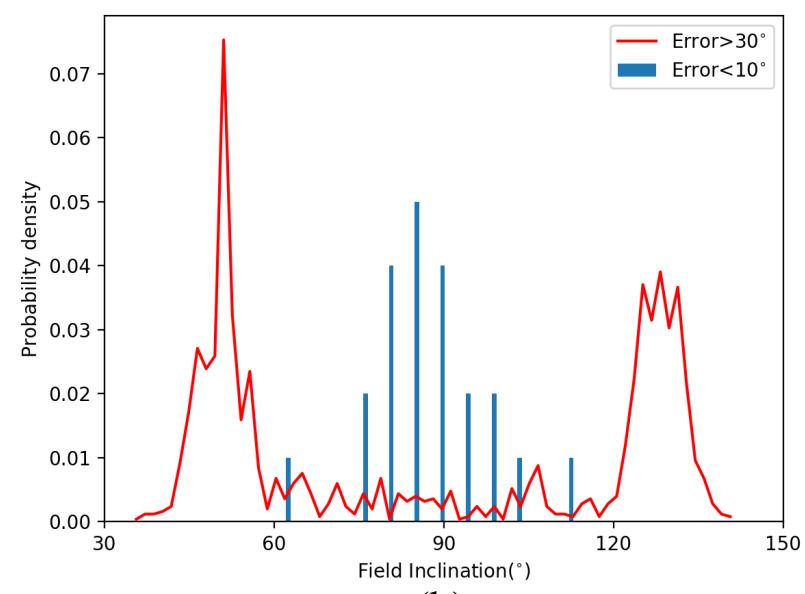

(b)

Fig. 4. THEMIS/MTR observations taken on September 24, 2013, showing $(a)$ the histogram of the magnetic strength for the full data set, and $(b)$ the histogram of the inclination angle of the magnetic field of the prominence.

It is interesting to note that for the inclination angle (Fig. 4b), these measurements have a peak around $85-90^{\circ}$, indicating that the field at these locations is mainly horizontal. There is however a wide scatter in the data.

In contrast, the red curves denote fits where the error in fitting the observed and model profiles exceeds $30 \%$, and the observed profiles therefore give a poor fit to the profiles of the model database. Consequently, the observed profile cannot be explained based on the model radiative transfer equations, which use a single unique magnetic field vector. For these data points the field strengths (Fig. 4a) lie in the range of 4-20 G. Throughout this range, the distribution is continuous, where fields in the range $12-20 \mathrm{G}$ occur with approximately equal probability. It is interesting to note that for these data points, the inclination angle (Fig. 4b) exhibits two peaks, one at about $50^{\circ}$ and the other at $130^{\circ}$. This shows that the deduced angle is not consistent with a horizontal field.

As discussed in Schmieder et al. (2014), the points with large error bars are not consistent with a single magnetic field value integrated along the line of sight. They are instead consistent with a more complex magnetic field configuration composed of a large-scale field along with a stochastic component. Such a stochastic component may be due to a number of physical reasons, and we note that stochastic does not necessarily mean turbulence. One possibility is that many small-scale unresolved plasma and magnetic field structures lie along the line of sight. When these structures are integrated over, they produce the observed data. Alternatively, another viable option is the presence of MHD turbulence. However, it is currently impossible to confirm whether either of these cases result in the observed profiles, or if a third physical mechanism is responsible.

Using the arguments presented above, Schmieder et al. (2014) concluded that the measured prominence fields were consistent with (i) a relatively weak but horizontal field in the range 5-15 G, which would represent the quasi-static large-scale field of the prominence (low error fits), and (ii) an additional region of stronger fields that include the background field along with a stochastic component (large error fits). It is important to note that the latter region existed at locations within the prominence where the most highly disorganised plasma motions occurred. For the present study, which considers the comparison of a static model with observations, we aim to determine whether the theoretical model can reproduce the inferred quasi-static background field alone (blue points with low error bars).

\section{Modelling}

To directly compare the observed magnetic field strengths in the prominence with the magnetic field deduced from a theoretical model, we chose to use the NLFF modelling technique of van Ballegooijen (2004). This technique was chosen because it uses photospheric magnetic field observations to construct the NLFF magnetic field configuration and thus can be directly compared to the limb observations. In addition, previous studies have shown that it can produce highly realistic representations of mid- to high-latitude filaments and prominences (see $\mathrm{Su} \&$ van Ballegooijen 2012; Su et al. 2015). Below we provide a brief description of the modelling technique (Sect. 3.1), followed by an analysis of the constructed magnetic field configuration (Sect. 3.2).

\subsection{NLFF modelling technique}

The NLFF field modelling technique applied within this paper is based on the technique that was first developed by van Ballegooijen (2004). Full details along with the development and successful application of this technique can be found in Bobra et al. (2008), Su \& van Ballegooijen (2012), and Su et al. (2015) and a brief description of the method is given below.

The NLFF magnetic field analysed in this paper was constructed in a spherical domain ranging from $\phi=\left[-5^{\circ}, 82.96^{\circ}\right]$, $\lambda=\left[-9.35^{\circ}, 61.52^{\circ}\right]$, and $r=\left[1 R_{\mathrm{s}}, 1.333 R_{\mathrm{s}}\right]$, where $\phi$ is the longitude, $\lambda$ is the latitude, and $r$ is the radius (measured in units of solar radii). Within this spherical domain, a uniform grid resolution of $0.115^{\circ}$ was used, where the number of grid points in longitude, latitude, and radial direction are 769, 769, and 145 . To produce an accurate model of the solar prominence observed on September 24, 2013 at the west limb, an accurate representation of the line-of-sight photospheric magnetic field underlying the filament on this date is required. Unfortunately, no direct observations of the photospheric field underlying the prominence exist on this date because photospheric magnetic field observations are currently only made along the Sun-Earth line. Beyond $\pm 60^{\circ}$ from central meridian, the measurement of the photospheric field rapidly loses accuracy. A proxy line-of-sight photospheric magnetogram was therefore constructed corresponding to the date of September 20, 2013, 12:00 UT. This is approximately four days before the passage of the filament across the limb. The model 
was constructed at this date and time because it provides the best balance between constructing the model as close as possible to the filament limb passage while maintaining the accuracy of the underlying line-of-sight photospheric field. Within the simulation domain, $\left(\phi=0^{\circ}, \lambda=0^{\circ}\right)$ corresponds to disc centre on September 20, 2013 at 12:00 UT.

To directly compare this with the observed field strength determined from the Stokes parameters and PCA inversion code described in the previous section, the magnetic field configuration constructed on September 20, 2013 at 12:00 UT was then rotated to the limb to match the observation date. We note that while the model is constructed on September 20, 2013 at 12:00 UT, the line-of-sight magnetogram we used is a composite of four SDO/HMI magnetograms. Each individual magnetogram of which the composite consists was taken at 12:00 UT between September 17-20, 2013. This is required in order to accurately represent the field near the limb, which cannot be observed. The magnetograms corresponding to September 17-19, 2013 were rotated to their corresponding longitudes on September 20, 2013 before they were combined with the observation taken on September 20, 2013. In the composite magnetogram the portions nearer the limb are dominated by the on-disc observations from September 17, 2013, (rotated to be at the limb), while the portions near central meridian are dominated by the SDO/HMI magnetogram from September 20, 2013. The composite line-of-sight magnetogram is shown in Figs. 5a and c. Previous studies such as those by Su \& van Ballegooijen (2012) and Su et al. (2015) have shown that the construction and use of a composite magnetogram as described above is the most advanced and effective way to minimise errors that are due to projection effects in the normal component magnetograms.

After we specified the line-of-sight field at the photosphere, we constructed the NLFF model of the filament and coronal field. Within the computational domain, the magnetic field $\boldsymbol{B}$ is expressed in terms of the vector potential $\boldsymbol{A}$, where $\boldsymbol{B}=\nabla \times \boldsymbol{A}$. To produce the NLFF model, a number of stages were applied as listed below.

1. A global Potential Field Source Surface (PFSS) model was constructed using the high-resolution composite SDO/HMI magnetogram for the region of interest, along with a lowresolution synoptic magnetogram for the remaining Sun.

2. The length, height, and path of the filament and its associated filament channel were determined by considering multiple observations, including $\mathrm{H} \alpha$, AIA 304, 171, $193 \AA$, and the HMI composite magnetogram.

3. From this, a 3D field-free cavity was created along this path by modifying the vector potentials of the potential field. After the cavity was produced, the coronal field was no longer potential and was no longer in force balance.

4. Axial flux $\Phi_{\mathrm{axi}}$ of a flux rope was inserted into the cavity along the observed path of the filament, which produced a thin flux bundle within the cavity. In addition, poloidal flux $F_{\text {pol }}$ may also be added to produce a twisted flux rope structure.

When this process was completed, the coronal field was no longer in equilibrium. To produce a new NLFF equilibrium, an iterative relaxation process was carried out for 70000 steps using the magneto-frictional relaxation technique (van Ballegooijen 2004). During this relaxation process, the line-of-sight photospheric field was held fixed, but reconnection was allowed to occur within the coronal volume as the inserted flux rope and overlying arcades interacted. This relaxation process continued until the magnetic pressure of the inserted flux rope balanced the tension of the overlying arcade. In general, we find that the final relaxed magnetic field configuration is not sensitive to the length or height of the 3D field-free cavity as long as appropriate values were used for quiescent and active region prominences (Bobra et al. 2008; Su \& van Ballegooijen 2012; Su et al. 2015). The final magnetic field configuration is instead more sensitive to the inserted axial and poloidal flux. In particular, the inserted flux rope rises and reconnects with the surrounding fields to reach an appropriate equilibrium. During this process, the flux rope may increase or decrease in length and change its shape.

Two separate model representations were constructed that we call model 1 and model 2, where the axial and poloidal fluxes were chosen to give stable magnetic field configurations. Model 1, which is shown in Fig. 5a, was constructed with $\Phi_{\text {axi }}=$ $2 \times 10^{20} \mathrm{Mx}$ and $F_{\mathrm{pol}}=2 \times 10^{10} \mathrm{Mx} \mathrm{cm}^{-1}$ and results in a twisted flux rope structure low down along the path of the filament, with overlying coronal arcades. In contrast, model 2 , which is shown in Fig. $5 \mathrm{c}$, was constructed with $\Phi_{\text {axi }}=2 \times 10^{20} \mathrm{Mx}$ and $F_{\mathrm{pol}}=0 \mathrm{Mx} \mathrm{cm}^{-1}$ and produces sheared arcades low down at the height of the filament. The two models represent the two distinct types of magnetic field configurations that are typically used in theoretical prominence models. In both cases, the filaments have a sinistral orientation that is of minority chirality to that of the northern hemisphere. For the remainder of the paper, our analysis is applied to the high-resolution domain covered by the composite HMI magnetograms.

\subsection{Analysis of field configurations}

In Figs. 5a and c we show representative field lines for the flux rope and the overlying arcade for (a) model 1 and (c) model 2 on September 20, 2013 12:00 UT. The magnetic field lines representing the filament take the form of an arc, where positive flux is located on the inner side of the arc and negative flux dominates around the outer edges. In general, the magnetic fields underlying the filament are weak. Figures $5 \mathrm{~b}$ and $\mathrm{d}$ show the same field lines for (b) model 1 and (d) model 2, where the entire magnetic field configuration has undergone a solid-body rotation to the date of September 24, 2013, 12:00 UT. This is close to the time where the prominence was observed at the limb. The solid-body rotation maintains the photospheric and coronal field distributions and was carried out using the rotation rate from Snodgrass (1983) calculated at $\lambda=35^{\circ}$, which corresponds to the middle latitude of the filament. In principle, a more complex differential rotation profile could have been applied, but because this first comparison is simple, the additional complexity involved is not necessary. From the limb view, the flux rope structure of model 1 (Fig. 5b) compared to the sheared arcade structure of model 2 (Fig. 5d) is very apparent.

In Fig. 6 we directly compare models 1 and 2 with AIA $193 \AA$ images. Panel (a) shows the AIA $193 \AA$ images on September 20, 2013 (left panel) and September 24, 2013 (right panel). The left-hand panel clearly shows the dark absorption feature of the filament on September 20, 2013, but it is difficult to identify any overlying arcades on this date. In contrast, when the prominence is at the limb on September 24, 2013, a series of almost concentric arcades lies above the prominence (see also Fig. 2a). In panel (b) the corresponding results are shown for model 1 , which is the strongly twisted flux rope solution. The left-hand panel clearly shows that on September 20, 2013, a twisted flux rope structure lies at the spatial location of the AIA $193 \AA$ filament. Within this panel, a series of arcade field lines is also plotted lying above the flux rope. In the right-hand image of panel (b) the field is rotated to September 24, 2013, 


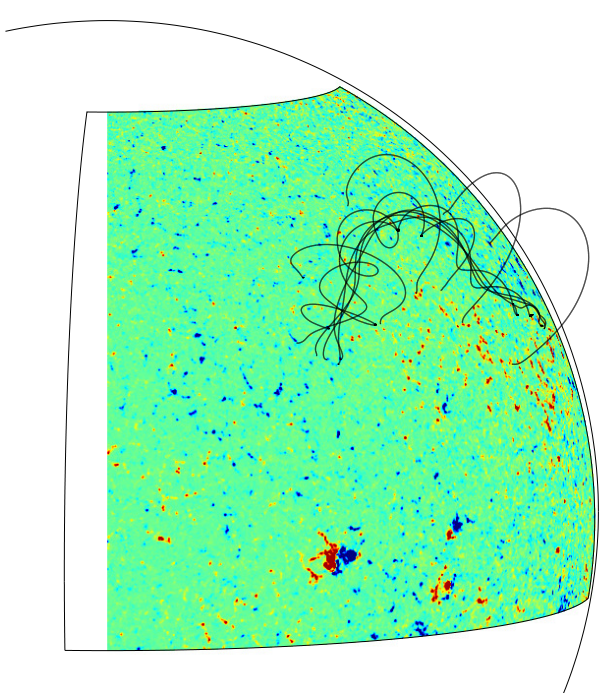

(a)

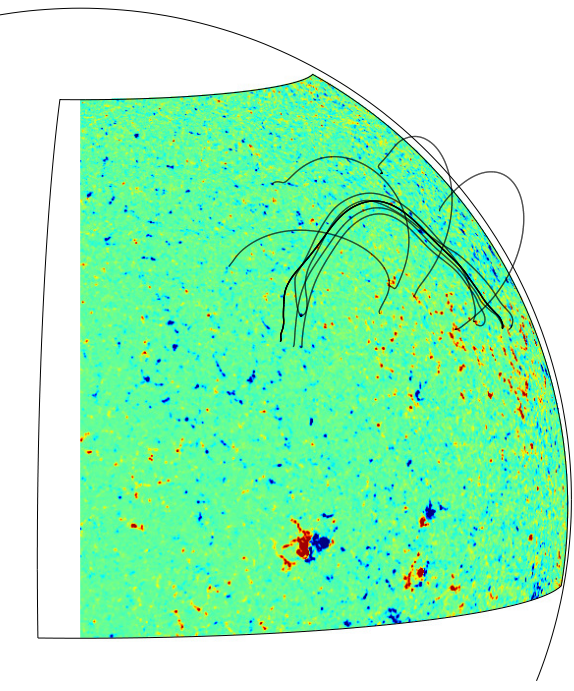

(c)

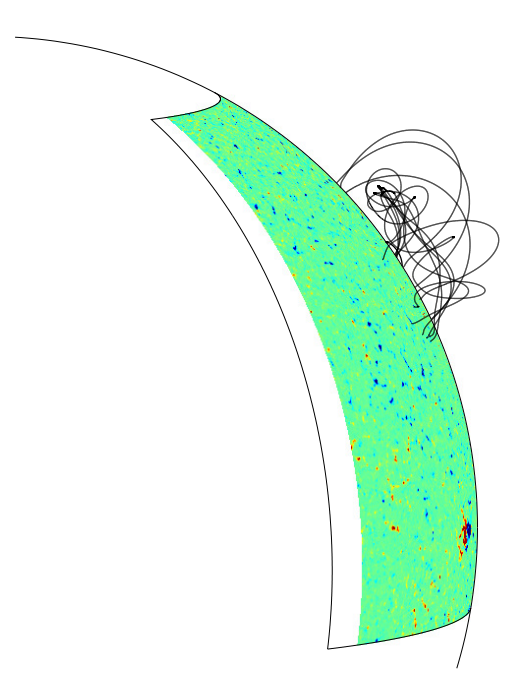

(b)

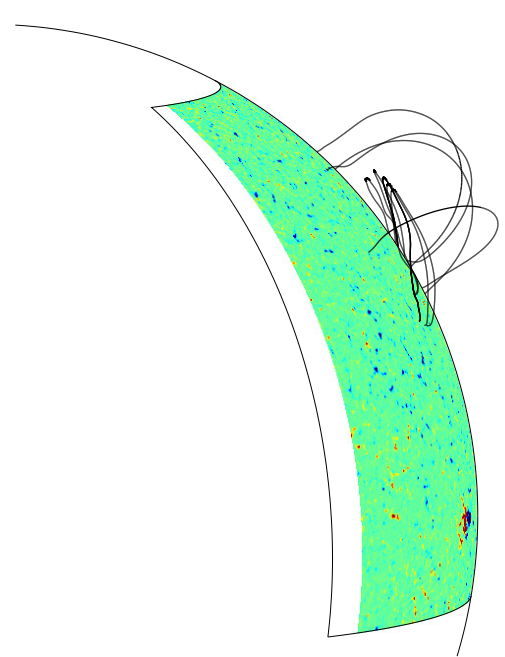

(d)
Fig. 5. Illustration of the NLFFF from the flux rope insertion model of van Ballegooijen (2004). Magnetic field constructed on September 20, 2013, 12:00 UT for $(a)$ model 1 and $(c)$ model 2 . The solidbody rotation of the constructed NLFFF to September 24, 2013, 12:00 UT is shown for $(b)$ model 1 and $(d)$ model 2. In each plot the thin black lines denote the solar limb and the area of the high-resolution computational box, and the thick black lines denote field lines. The colour image illustrates the photospheric magnetic field distribution. Red and blue denote positive and negative flux. where the arcade field lines produce a series of concentric field lines above the flux rope structure. These field lines match the height and width of the loops in AIA $193 \AA$ well. Finally, panel (c) shows the same results, but for model 2 , the sheared arcade solution. When the results for September 20, 2013 (left panel) are compared with those of model 1 , model 2 produces a slightly poorer agreement with the path of the AIA $193 \AA$ filament. While this is the case, the differences are small. The right-hand image of panel (c) shows that model 2 also has a series of concentric field lines lying above the sheared arcade, which again match the AIA $193 \AA$ coronal loops well. This clearly shows that models 1 and 2 produce a good agreement with the path of the filament and the overlying AIA $193 \AA$ coronal loops. Because both models compare equally well to the overlying loops, the loops cannot be used to distinguish between the models. We now compare this with the $\mathrm{HeD}_{3}$ observation from THEMIS/MTR.

Care must be taken when observations of the magnetic field in solar prominences are compared to the magnetic fields obtained in the computational models. Direct measurements of the observed prominence magnetic field are carried out in the $\mathrm{He} \mathrm{D}_{3}$ line, which originates in the prominence plasma. Because the constructed NLFF models do not contain any plasma, we need to determine in principle which locations within the constructed field may support cool plasma against gravity. To determine these locations, we considered dips in the magnetic field lines. To identify dips in the spherical coordinate system, the strict mathematical condition is

$B_{\mathrm{r}}=0$

and

B. $\nabla B_{\mathrm{r}}>0$.

While this is the strict condition, it is unlikely because of the nature of the finite discretisation of the computational model that any grid points identically satisfy Eq. (1). We therefore replace Eq. (1) with the condition

$\frac{B_{\mathrm{r}}}{B_{\mathrm{h}}} \leq 0.15$,

where $B_{\mathrm{h}}=\sqrt{B_{\theta}^{2}+B_{\phi}^{2}}$ is the local horizontal field in the spherical coordinate system. Equation (3) identifies all grid points 

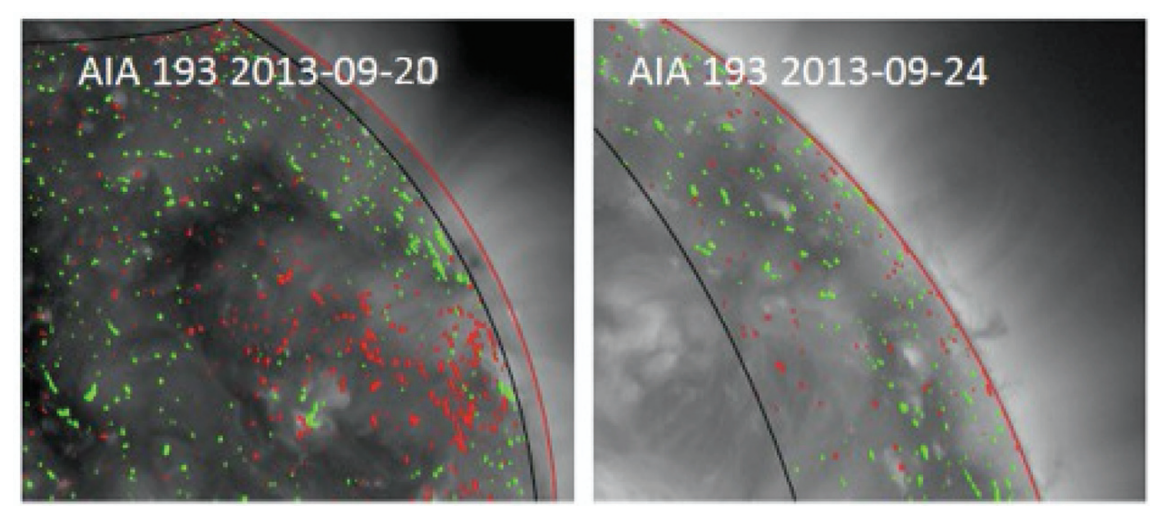

(a)
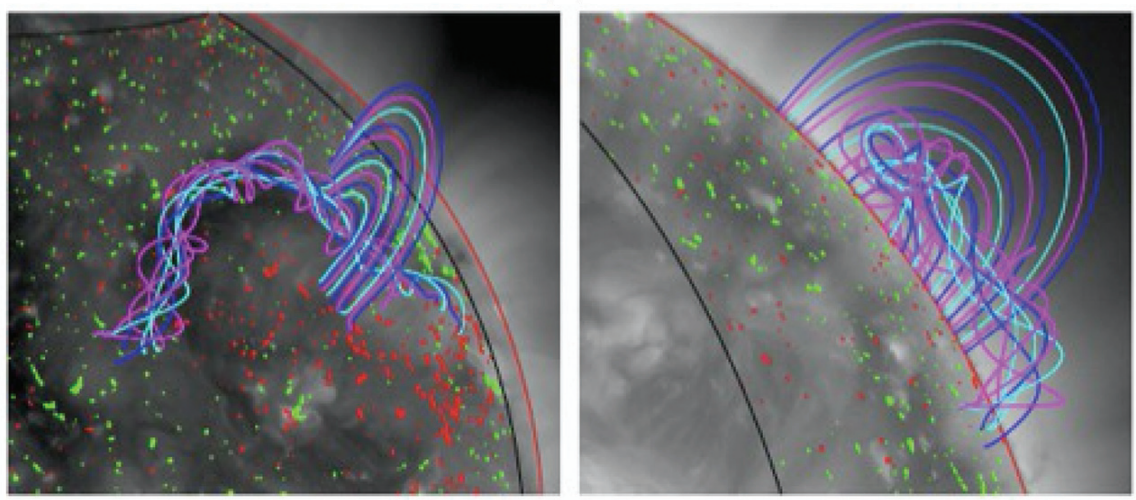

(b)
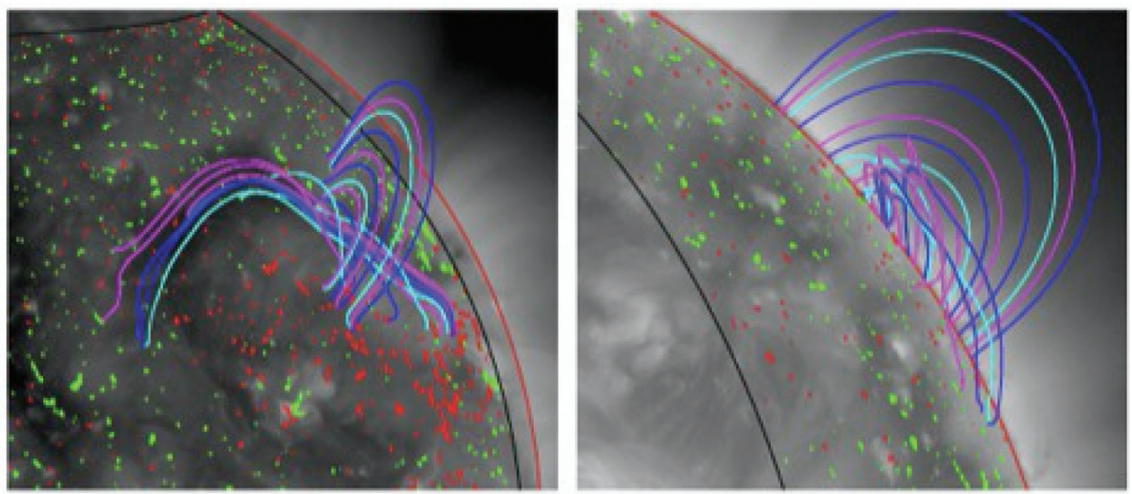

(c)
Fig. 6. Comparison of (a) AIA $193 \AA$ images with (b) model 1 and (c) model 2 for September 20, 2013 (left column) and September 24, 2013 (right column). In each image the red line denotes the solar limb, and the black line denotes the computational domain. In all of the images the underlying photospheric magnetic field is shown as the red and green contours. where the local field vector makes an angle smaller than $8.5^{\circ}$ to the local horizontal. This relaxed condition (combined with Eq. (2)) is used because in Fig. 4 the low error bar points (blue bars) do not take the form of a delta function located at $90^{\circ}$, but rather a distribution around it. In Eq. (3) the condition has been varied from 0.1 to 0.25 without any significant change in the results.

Only grid points that satisfied conditions (2) and (3) in the spherical coordinate system were retained in models 1 and 2, where any grid points that did not satisfy these conditions had their field strength set to zero $(\boldsymbol{B}=0)$. We assumed for our comparison that each identified dipped grid point contributed equally to the line-of-sight integration of the magnetic field. This is similar to assuming that each dip has the same density weighting in the low plasma- $\beta$ approximation along with an optically thin assumption. The low plasma- $\beta$ condition means that the plasma does not alter the field strength or shape of the magnetic field. In principle, a more advanced form of modelling could mass-load field lines and then compute the emission in $\mathrm{He}_{3}$, to reconstruct the field strengths. However, if these calculations were carried out using an optically thin assumption where the same density weighting is applied to each dip, it would just reproduce the field values that are already identified at each dip. This approach may only produce different results if either statistical filling of dips with varying plasma density or optically thick radiative transfer models are applied. Approaches like this are far beyond the scope of the present paper because they would require the development of an optically thick radiative transfer model for $\mathrm{He}_{3}$. For the following analysis, we therefore analyse the field strengths in the identified dipped grid points where each dipped grid point is given an equal weighting.

The distribution of the dips as a function of longitude and latitude is shown in Fig. 7 at various radii for (a)-(c) model 1 and (d)-(f) model 2. The longitude coordinates in this plot are given 


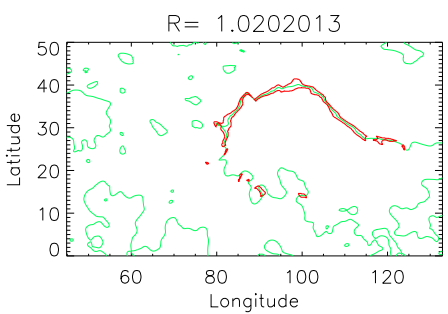

(a)

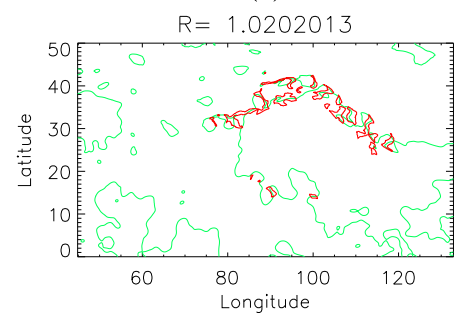

(d)

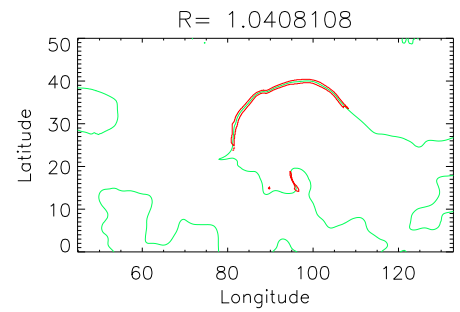

(b)

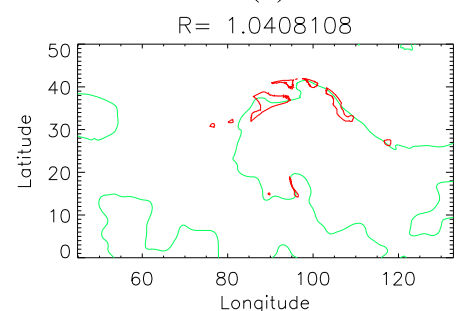

(e)

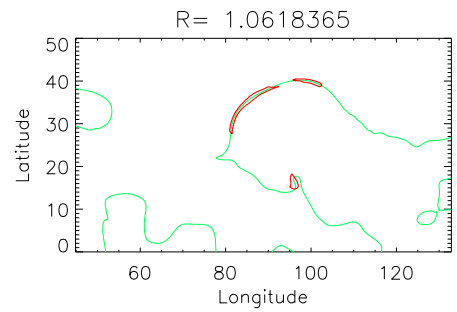

(c)

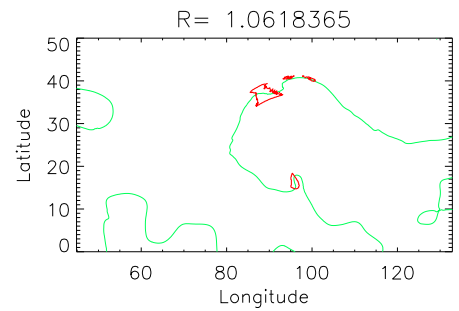

(f)

Fig. 7. Locations of dips for model $1(a)-(c)$ and model $2(d)-(e)$, shown at radial distances of $(a)$ and $(d) 1.02 R_{\odot},(b)$ and $(e) 1.04 R_{\odot}$, and $(c)$ and $(f) 1.06 R_{\odot}$. In each plot the red contour denotes the location of the dips, and the green contour shows the location of the PIL at each radial distance. The longitude and latitude are expressed in terms of the rotated values of the model for 12:00 UT on September 24, 2013.

in terms of the rotated coordinates corresponding to September 24, 2013, 12:00 UT. The plots are shown for (a) and (d) $r=$ $1.02 R_{\mathrm{s}}$, (b) and (e) $r=1.04 R_{\mathrm{s}}$, and (c) and (f) $r=1.06 R_{\mathrm{s}}$, where in each case the green lines denote the PIL at the respective radial distance and the red contour denotes the location enclosed by dips in the field.

In models 1 and 2 , the dips show a very fragmented structure below $r=1.01 R_{\mathrm{S}}$ (not shown) that is due to weak mixed polarity fields that lie close to the PIL. Figure 7 clearly shows that model 1 with the stronger poloidal flux produces a much more coherent large-scale pattern of dips at each of the radii. In particular, the dips form a continuous band along the full arc of the PIL from $\phi=80^{\circ}$ to $\phi=120^{\circ}$. In contrast, model 2 shows a much more fragmented form. In models 1 and 2 the extent of the dips along the PIL decreases with height. However, in models 1 and 2 the dips extend to a similar height of $1.083 R_{\odot}(58000 \mathrm{~km})$.

At this point in the analysis, it is important to recognise that filaments and prominences may look very different and extend over different spatial scales depending on the wavelength used and whether they are viewed as a filament or prominence. This can be due to the specific emission or absorption properties of the spectral line in addition to the dependence of the intensity of the line on the plasma density and the line-of-sight integration. Within the model the height of the dips extends to $58000 \mathrm{~km}$. However, the observed prominence in $\mathrm{H} \alpha$ and $\mathrm{He} \mathrm{D}_{3}$ extends to just over $26000 \mathrm{~km}$ when seen on the limb. The difference in physical extent may be due to a number of reasons. One possibility is that the dips become shallower with height, which results in less dense plasma and correspondingly less emission. Gunár et al. (2014) and Gunár \& Mackay (2015a,b) have shown that when mass-loaded dips are viewed either in absorption or emission, this can lead to a different physical extent and morphology of the dips. Moreover, at any single time, not all of the dips within the theoretical model need to contain plasma.

Figures $8 \mathrm{a}$ and $\mathrm{b}$ show filled contour plots for the number of grid points that are classified as dipped along the line of sight. The transition from black to white corresponds to 1-60 data points, where the exact colour coding is given by the bar on the right-hand side. No units are given because the quantity is dimensionless. In each of the plots the solar limb is shown by the dashed line, and the dotted lines give the position angles at $290^{\circ}$ and $312^{\circ}$, respectively. The black dashed box denotes the field of view of the THEMIS/MTR observations. For models 1 and 2 it is clear that the radial and latitudinal extend of the dips exceeds the THEMIS/MTR field of view. Models 1 and 2 have a continuous series of dips throughout the THEMIS/MTR field of view. A common feature of both models is that more dipped grid points are found in the northern half of the THEMIS/MTR field of view, where the line of sight direction is along the east-west extension of the prominence. Correspondingly, as the prominence transitions to north-south near the southern end of the THEMIS/MTR field of view, the number of dipped grid points along the line of sight decreases rapidly as we look perpendicular to the prominence axis. It is interesting to note that the spatial location of maximum intensity in $\mathrm{He} \mathrm{D}_{3}$ corresponds to the upper range in the number of grid points summed along the line of sight. For models 1 and 2 the isolated region of dips close to the position angle of $285^{\circ}$ is present as a result of the underlying field configuration that naturally produces dips in the initial potential field. It is not a consequence of the injected axial or poloidal flux.

\section{Comparison of observations and models}

\subsection{Qualitative comparison}

Figures 9a and $\mathrm{b}$ show the line-of-sight average of $|\boldsymbol{B}|$ determined from the grid points that are classified as dipped for (a) model 1 and (b) model 2 . We calculated the line-of-sight average by considering only points that are within $\pm 10^{\circ}$ of the limb to take the portions of the prominence into account that would be visible above the limb as a result of the curvature of the Sun. This simple approach to the average was taken because this first attempt at a direct comparison has many sources of error, which are discussed in Sect. 5. For both cases, a filled contour plot of the line-of-sight average of $|\boldsymbol{B}|$ is shown for $1-11 \mathrm{G}$, where the colour scale is given by the bar on the right-hand side. The scale transition from black to white denotes increasing field strengths. For the plots in Fig. 9 a zoomed-in view compared to Fig. 8 is shown such that the area enclosed by the white dashed box can be seen. This box represents the THEMIS/MTR field of view. Once again the black dashed line denotes the limb of the Sun. In 


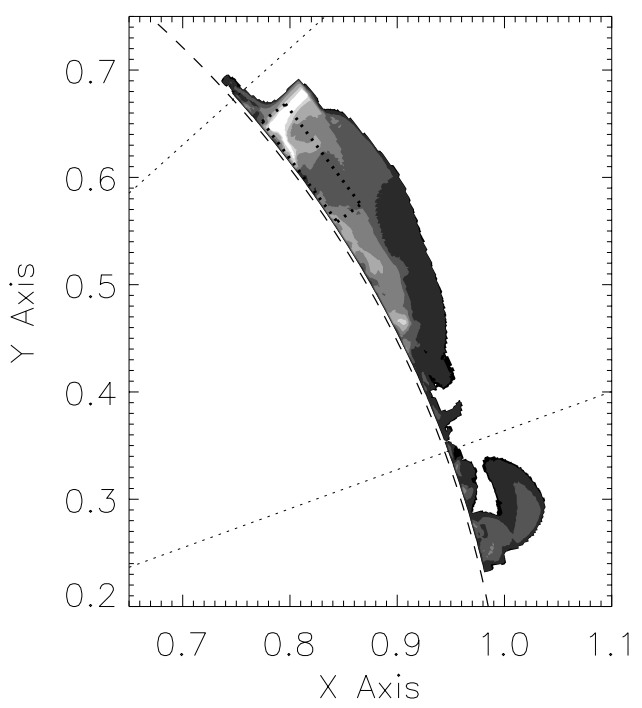

(a)
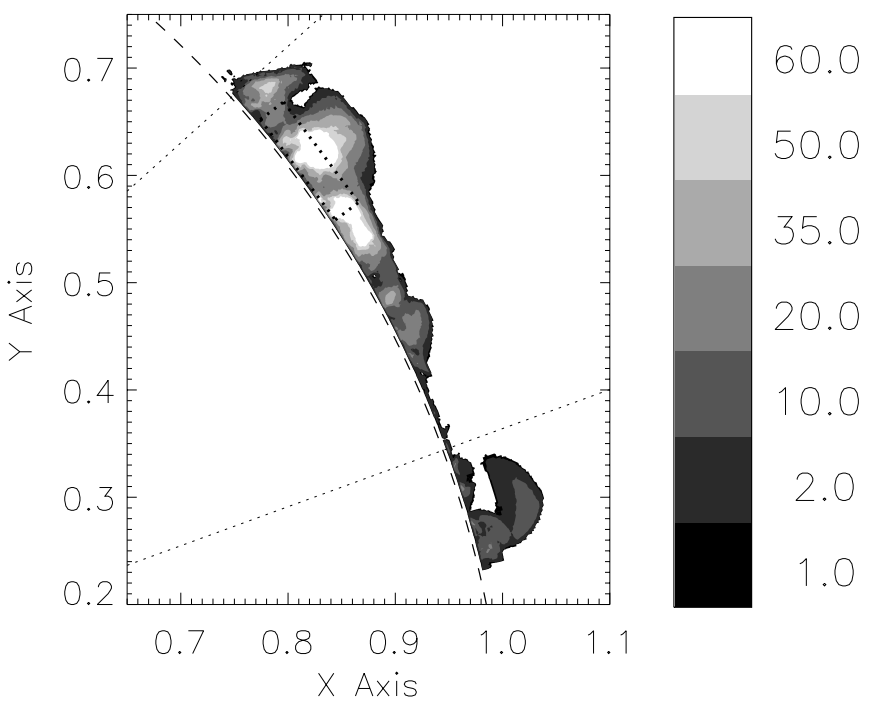

(b)

Fig. 8. Filled contour plots for the number of dipped grid points along the line of sight for $(a)$ model 1 and $(b)$ model 2. In each plot the solar limb is shown by the dashed line, where the dotted lines denote the position angles at $290^{\circ}$ and $312^{\circ}$. The black dashed box denotes the THEMIS/MTR field of view. For each of the contour plots the colour bar at the right-hand side gives the contour levels for the number of grid points.

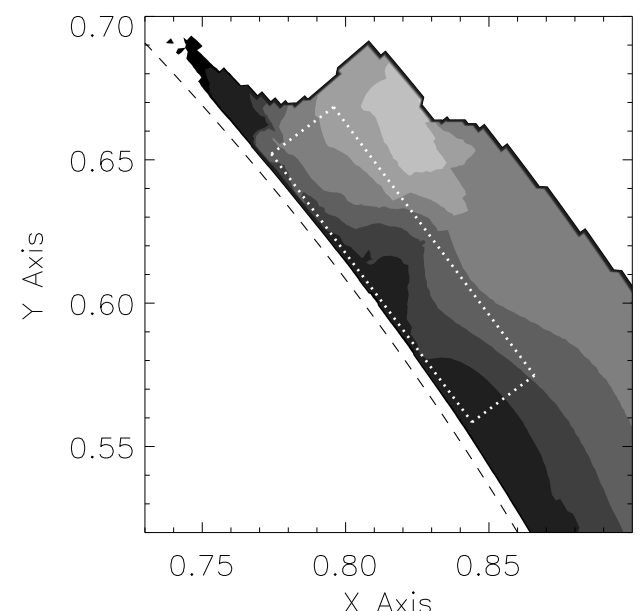

(a)

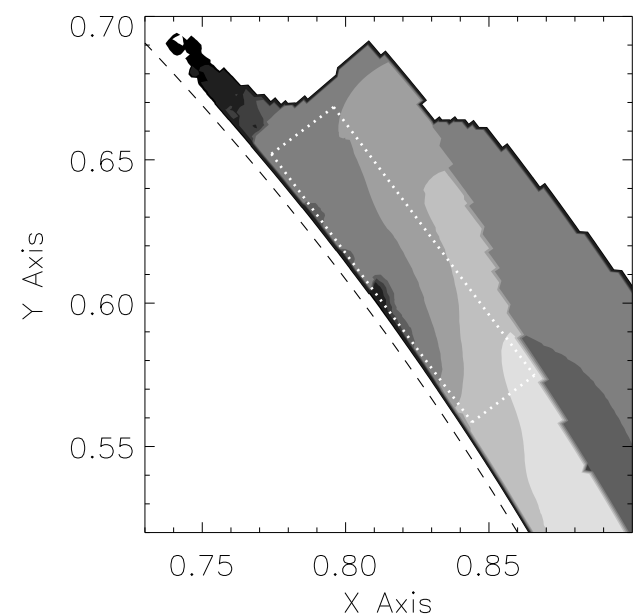

(c)

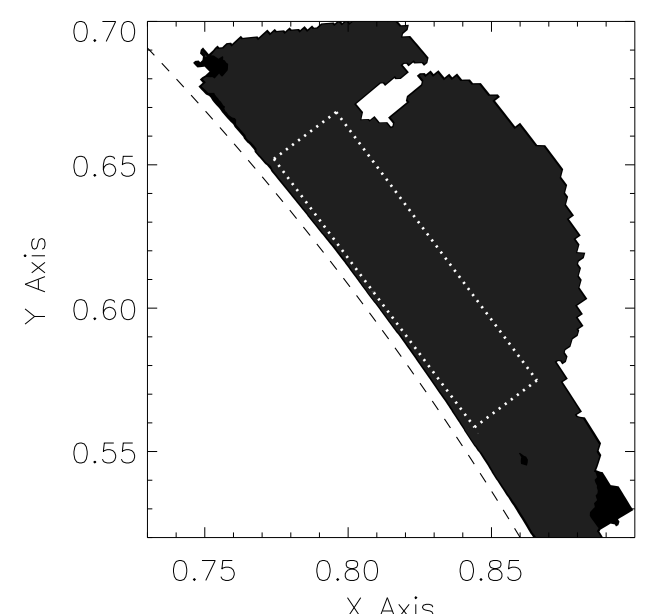

(b)

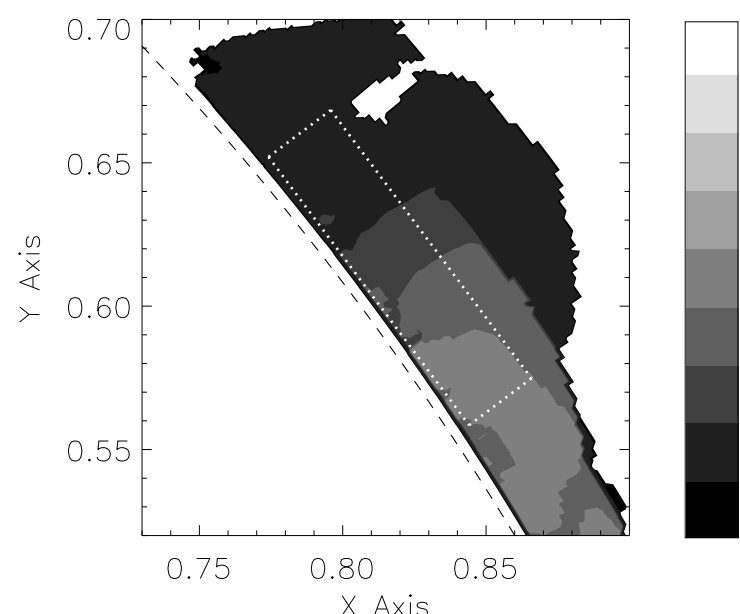

(d)

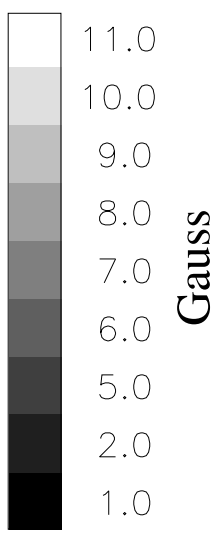

Fig. 9. Properties of the dipped magnetic field regions for model 1 (left column) and model 2 (right column). In each plot the solar limb is shown by the dashed line. $a$ and $b$ : filled contour plots for the line-of-sight average of $|\boldsymbol{B}| . c$ and $d$ : filled contour plots for the peak value of the magnetic field along the line of sight. For each of the contour plots the colour bar at the right-hand side gives the contour levels. 
each plot the dipped region clearly extends to nearly twice the radial extent of the THEMIS/MTR field of view. In addition, the dipped region is nearly twice the latitudinal extent. It is interesting to note that in Fig. $3 \mathrm{~b}$ over the full extent of the box, the computed field values do not drop to zero at the top of the box. This indicates that the THEMIS/MTR field of view does not capture the full radial extent of the filament channel or prominence. Therefore any theoretical model that yields an extended region of dips beyond the THEMIS/MTR field of view is not inconsistent with the THEMIS/MTR observations. We list the key similarities and differences from a comparison of models 1 and 2 with Fig. $3 \mathrm{~b}$ below.

- Model 1 exhibits much higher line-of-sight averaged field strengths than model 2 . In model 1 the dipped field extends over a much wider range of latitudes.

- The highest value for the line-of-sight averaged field strength in model 2 is below $5 \mathrm{G}$, which is far too low to match the values found in Fig. 3b. There is also weak spatial variation throughout the length of the prominence.

- Model 1 exhibits line-of-sight averaged field strengths over the full range of values. The largest variation is found with radial distance across the prominence. An increasing magnitude with radial distance is required for the presence of magnetic dips.

- Models 1 and 2 produce a smooth variation of the field strengths with much less small-scale variation than the observations.

- Near the northern end, field strengths of 7-9G are found in model 1. This location is consistent with the region denoted by the oval in Fig. 3b, where relatively uniform field strengths of 8-10 G were observed.

- In the middle to southern end of model 1 the field strengths lie in the range $5-7 \mathrm{G}$. These values are too low by a factor of about 2 to match those found in Fig. $3 b$.

The itemised points clearly show that when the line-of-sight average of $|\boldsymbol{B}|$ is considered, model 1 produces a much better match than model 2. However, this match is not perfect: a difference of a factor of 2 in the field strengths occurs at the southern end of the prominence. It is interesting to note that at the northern end of model 1, which produces field strengths consistent with those found in Fig. 3a, the highest number of grid points is used to construct the average. This is also the spatial location where the observations show the least spatial variation in field strength.

As an alternative way of representing the properties of the prominences magnetic field, Figs. 9c and d show a similar plot, but now for the peak field strength along the line-of-sight. It is important to note that the filled contour plot is now shown for higher values of the field strength, where the values range from $1-15 \mathrm{G}$. The bar at the right-hand side again gives the values. The key results are listed below.

- Model 2 again produces field strengths that are too low to match the observations.

- At the northern end of model 1, field strengths of 7-9 G are again obtained. This is consistent with those found in the oval region in Fig. 3a.

- In model 1, as the prominence transitions southward, the peak field strength increases. This is consistent with the observations. At the southern end, an extended region of around $11-13 \mathrm{G}$ occurs. These values are consistent with the values found outside of the oval in Fig. 3, but they occur at a larger radial height in the model.

This discussion shows that model 1 again produces a much better match than model 2. This time the results are more consistent with the observations.
When we compare the results from Figs. 9a and c, it is interesting to note that the peak and average values give similar results when we look along the prominence axis (east-west portion of the filament) where the average is calculated over a large number of data points. Both representations of the field in this portion of the prominence produce field strengths that are consistent with the observations. In contrast, when we look perpendicular to the prominence axis (north-south portion), the average values along the line of sight are much lower and less consistent with the observations. In contrast, the peak values along the line of sight produce results that are more consistent with the observations. At these locations, the average is taken over the fewest data points. While it needs further investigation for a conclusive answer, this suggests that the observed profiles in $\mathrm{He} \mathrm{D}_{3}$ might illustrate different properties of the field depending on the depth of integration across the prominence: for small depths of the line-of-sight integration, peak values of the magnetic field may dominate along the line of sight.

\subsection{Quantitative comparison}

We now consider a quantitative comparison of the strength of the magnetic field obtained from the observations with that deduced from model 1 and model 2 . In carrying out the comparison, it should be noted that the spatial resolution of the theoretical model is much higher than that of the observations. As a consequence, the theoretical data are expected to produce a smoother and more continuous distribution. Figure 10 shows the probability distribution of the field strengths at the dipped locations for (a) and (c) model 1 and (b) and (d) model 2, where Figs. 10a and $\mathrm{b}$ give the results for the average value of the magnetic field along the line-of-sight and Figs. 10c and d for the peak value along the line-of-sight. The distributions of the field strength were only computed for locations that lie within the THEMIS/MTR field of view. In line with Fig. 4, the plots were normalised such that the area below each curve was set to one. Because the observational data we compared our result to have a less continuous distribution and the cut-off values are different, care must be taken in comparing the distributions because each distribution is normalised. We therefore chose to compare the peak locations in the distributions and not their size or relative size because the size may be influenced by the continuous or non-continuous nature of each data set, and cut-offs when present.

Figure 10 shows that model 1, which has the higher poloidal flux, exhibits higher field strengths and a wider range of values than model 2. When we compare Figs. 10a and b with the blue bars in Fig. 4a, model 1 clearly produces a better agreement with the observations than model 2 . The dominant peak of the field strength in model 2 lies at about $4 \mathrm{G}$, which is too low by about a factor of 4 compared to that found in the observations. Model 2 also contains a negligible number of data points where the field strength is greater than $5 \mathrm{G}$. In contrast, the dominant peak for model 1 is at around $7 \mathrm{G}$, which is only a factor of 2 too low to match the observations.

A much better agreement with the observed probability distribution occurs when we consider the peak value of the field strength along the line-of-sight (Figs. 10c and d). The distribution of the field values in model 1 covers a much wider range than in model 2 . In model 1 non-negligible values occur up to $13 \mathrm{G}$, while this value is much lower at $8.5 \mathrm{G}$, for model 2. Model 2 again produces field strengths that are too low. Model 1 shows a clear peak at $8.5 \mathrm{G}$ with non-negligible values up to $13 \mathrm{G}$. It is also interesting to note that model 1 produces a lower cut-off 


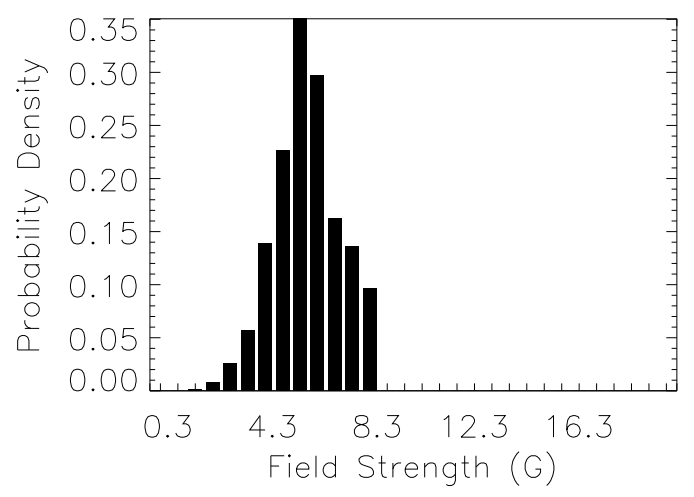

(a)

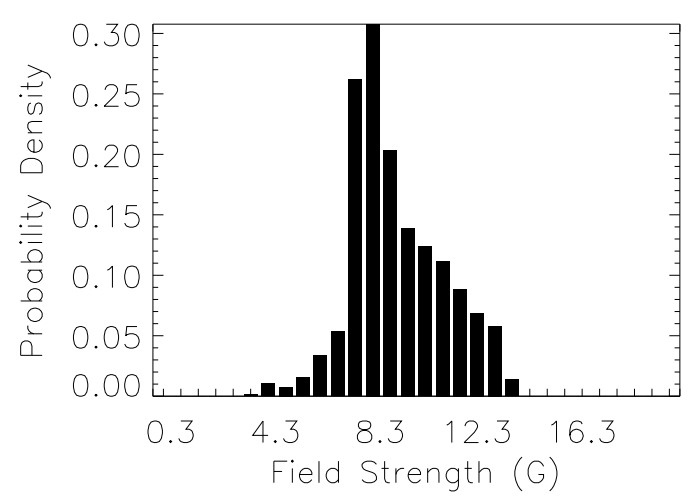

(c)

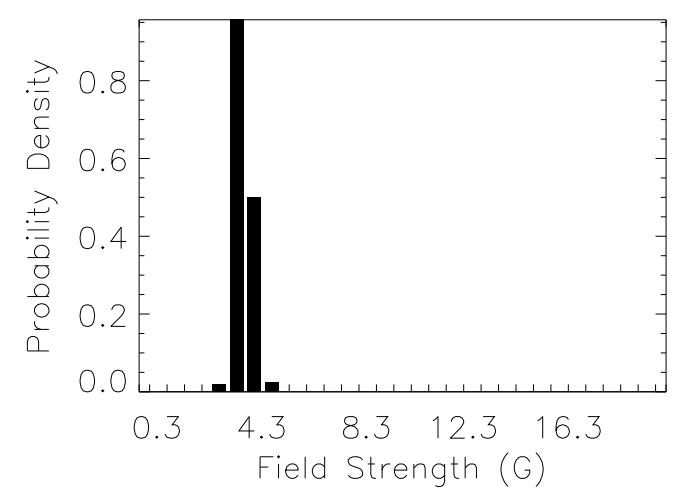

(b)

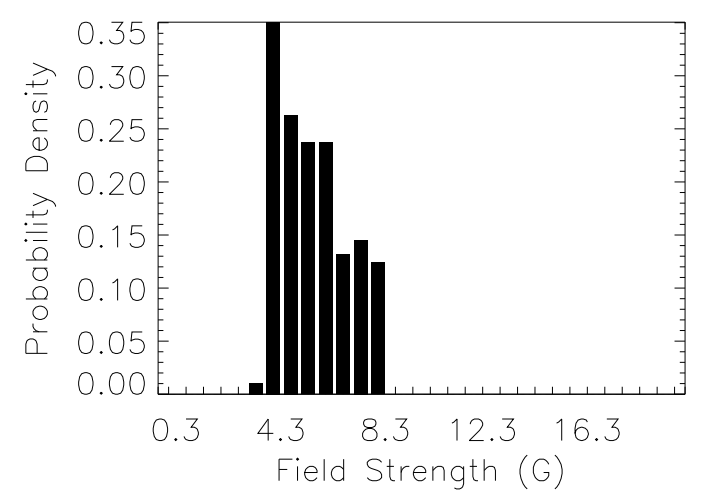

(d)

Fig. 10. Normalised distributions for the magnetic field in magnetic dips for model 1 (left column) and model 2 (right column). The distributions are shown for $(a)$ and $(b)$ the line-of-sight average of $|\boldsymbol{B}|,(c)$ and $(d)$ for the peak value of $|\boldsymbol{B}|$.

just below $7 \mathrm{G}$, where very few data points have values below this. This cut-off level matches that found in the observations, where the cut-off in model 2 is much lower. This discussion clearly shows that the distribution obtained from model 1 for the peak field strengths produces the best match to the observations. Distributions of the inclination angle for the dipped field lines in models 1 and 2 were not included: as a result of the identification and selection criteria for the dips that are described in Sect. 3.2, they both produce a distribution centred at $90^{\circ}$ with all inclinations within $\pm 8.5^{\circ}$.

\section{Conclusion}

We have carried out the first direct comparison of magnetic field measurements taken in a prominence with those deduced from a theoretical model. Understanding the nature and properties of the magnetic field that threads through a prominence is key to understanding how prominences may erupt and subsequently influence space weather. High resolution magnetic field measurements within filaments or prominences are not routinely made, therefore it is important to test whether models can produce realistic results and in which parameter regimes these results occur. If quick and efficient data -constrained models are found to produce a realistic representation of the strength and distribution of magnetic fields in prominences, such tools will be a powerful technique for operational space weather warnings. In particular, they will provide valuable information on the cores of coronal mass ejections, which are often related to filaments or prominences. As a first step, we tested the NLFFF model of van Ballegooijen (2004) with the magnetic field deduced from THEMIS/MTR He $\mathrm{D}_{3}$ observations.
From September 20-24, 2013, the transition of a large Ushaped filament on the solar disc in the northern hemisphere to a prominence at the west limb of the Sun was followed. The filament was composed of two distinct parts: an east-west section lying at nearly constant latitude, and a north-south section spanning a range of latitudes. Schmieder et al. (2014) discussed the dynamics and magnetic field of the prominence on September 24, 2013 in detail. The THEMIS/MTR observations in the $\mathrm{He}_{3}$ line that we used to determine the observed properties of the magnetic field of the prominence (Figs. 3 and 4) produced two distinct data sets. The first data set with error bars lower than $10 \%$ (blue distribution in Fig. 4) represents a good fit to the theoretical profiles produced from the $\mathrm{He} \mathrm{D}_{3}$ polarised radiative transfer equations. For these points, the deduced magnetic field properties can be regarded as reliable; magnetic field strengths in the range 8-19 G were obtained where the field was mainly horizontal. In contrast, for the second data set, the error was greater than $30 \%$ (red distribution in Fig. 4). For this data set, the observed profiles in $\mathrm{He}_{3}$ did not fit the database of theoretical profiles, and the values were therefore deemed unreliable. It is interesting to note that these data points did not produce a horizontal field. Because the interpretation of the latter data set with large errors is unclear, the data set was excluded from the comparison with the quasi-static model. The spatial distribution of the magnetic field shown in Fig. 3 also produced two distinct areas. At the northern end of the prominence, denoted by the oval, the field was mainly between 8 and $10 \mathrm{G}$ and had a weak spatial variation. In contrast, stronger fields $(15 \mathrm{G})$ with a stronger spatial variation were found within the southern projection of the filament.

A major difficulty to overcome in order to carry out the comparison was that direct magnetic field measurements of 
prominences only occur at the limb of the Sun. In contrast, data-constrained theoretical models require photospheric magnetograms that can currently only be taken along the Sun-Earth line. This means that we have no direct observations of the distribution of the photospheric magnetic field that underlies the prominence at the time when its magnetic field is measured. To overcome this problem, we chose to use the data-constrained non-linear force-free model of Su \& van Ballegooijen (2012) and Su et al. (2014). In this approach a composite normal component magnetogram was constructed from daily SDO/HMI magnetograms covering September 17-20, 2013 to minimise the errors due to projection effects in the normal component magnetogams near the limb. This composite magnetogram is regarded as the most advanced and best possible representation of the underlying photospheric field at the time that the THEMIS/MTR observations were made.

To construct the composite normal component magnetogram for the lower boundary condition in the NLFF field model, four daily SDO/HMI magnetograms were taken between September 17-20, 2013. Portions that were not significantly affected by fore-shortening were extracted, and a composite produced corresponding to the time and date of 12:00 UT on September 20, 2013. Within this composite magnetogram (Fig. 5), portions closer to central meridian are dominated by the SDO/HMI magnetogram taken on September 20, 2013, while those closer to the limb were dominated by the on-disc magnetogram of September 17,2013 , rotated to the correct longitudes closer to the limb corresponding to September 20, 2013. From this composite normal component magnetogram, a potential field was constructed into which a magnetic flux rope was inserted following the procedure of van Ballegooijen (2004). The physical dimensions (length and height) of the inserted flux rope were deduced from studying a combination of $\mathrm{H} \alpha$, AIA 304, 171, $193 \AA$, and magnetogram observations. Two separate NLFF field solutions were then constructed for which the axial and poloidal flux of the inserted flux rope were chosen to give stable non-eruptive solutions after relaxation to a NLFF field. For the two computed magnetic fields, which we called model 1 and model 2 , the axial and poloidal flux of the inserted flux ropes was $\Phi_{\mathrm{axi}}=2 \times 10^{20} \mathrm{Mx}$, and $F_{\mathrm{pol}}=2 \times 10^{10} \mathrm{Mx} \mathrm{cm}^{-1}$ for model 1 and $\Phi_{\text {axi }}=2 \times 10^{20} \mathrm{Mx}$ and $F_{\text {pol }}=0 \mathrm{Mx} \mathrm{cm}^{-1}$ for model 2 . Both models produced a good representation of the path of the observed $\mathrm{H} \alpha$ filament on September 20, 2013 (Fig. 5). The key difference between these two solutions was that model 1 produced a twisted flux rope structure along the path of the observed filament, while model 2 resulted in a sheared arcade structure. Thus the two models represented the two types of magnetic field configurations that are commonly used to represent filaments or prominences (Mackay et al. 2010). While these models were constructed on September 20,2013, when the prominence was still a filament on the solar disc, this was 4 days before the THEMIS/MTR magnetic field measurements were made at the limb. To place the magnetic field configuration at the limb, a solid-body rotation was applied. This may have introduced a source of error that is discussed below.

To compare the theoretical models to the observations, grid points within the theoretical models that contained dips were identified and the magnitude of the field determined. We determined a line-of-sight average of the magnetic field strength and its peak value along the line of sight. These two quantities were then compared to the observations. This comparison clearly showed that model 2, which was the sheared arcade structure with less twist, gave a poor agreement to the observations. The line-of-sight average produced field strengths that were more than four times to low, while for the peak value, the results were too low by about a factor of 2 . In contrast, model 1 , the twisted flux rope solution, produced a much better agreement. For its line-of-sight average, the results were now too low by a factor of 2. However, poor agreement was only found for the region outside of the oval in Fig. 3b. This corresponded to the northsouth extension of the filament, where the integration was across, rather than along, the filaments axis. Inside the area denoted by the oval, where the integration was along the filaments axis, an average field strength of $7-9 \mathrm{G}$ was found. This agreed well with the observations. The comparison between model 1 and the observations significantly improved when the peak value along the line of sight was considered. Good agreement was again found at the location of the oval, where the peak values and the line-of-sight integration gave approximately the same values, but now at the southern extension of the prominence, model 1 produced field values in the range $11-13 \mathrm{G}$, which is only a few Gauss lower than those found in the observations, $\sim 13-15 \mathrm{G}$. Because model 1 produced the best comparison with the observations, we conclude that the observed magnetic field configuration of the prominence is that of a flux rope. This result is consistent with the study of Ouyang et al. (2017), who found that $96 \%$ and $60 \%$ of quiescent and active region filaments are supported by flux ropes. While model 1 clearly produced results that were far more consistent with the observations, models 1 and 2 both produced field configurations that were much smoother than the configuration found in the observations. However, because of the potential errors in the comparison, this difference is to be expected. It is interesting to note that for the line-of-sight average and the peak values along the line of sight, the best agreement between model 1 and the observations is found when the comparison is made along the flux rope axis.

Several sources of potential error in our comparison are related to the use of the theoretical model and the observations. For the theoretical model, key sources of error are related to the NLFF field modelling approach, the construction of a composite magnetogram, and the fact that the model is constructed four days prior to limb passage and the THEMIS/MTR observations. The latter two sources of error, which correspond to a time difference between the model and observations, are currently difficult to overcome without a magnetograph placed either at the L4 or the L5 point (Mackay et al. 2016). While there will have been a number of changes to the small-scale photospheric field along the PIL during the four days because of the mid- to high latitude location of the filament and because the filament structure remained relatively stable, these will probably not have had a significant effect on the magnitude of the field obtained in the model. Moreover, limb observations did not indicate any large-scale flux emergence or the appearance of new loop structures. Finally, from the modelling perspective, to consider any potential errors from the NLFF field modelling approach, future studies should consider alternative MHD approximations. These might include magnetohydrostatic models (Aulanier \& Démoulin 2003) or full MHD solutions (Lionello et al. 2002). Another source of error in the modelling arises from the assumption that each grid point that contains a dip has an equal weighting for the magnetic field strength along the line of sight. To remove this approximation, statistical filling of dips with varying plasma density would be required along with developing a cross field plasma structure. However, such an approach would only produce significantly different results if an optically thick radiative transfer model for $\mathrm{He}_{3}$ were applied. The development of 
such a model is far beyond the scope of the present paper but should be considered in the future.

Several sources of error also lie within the observations and the process we used to reduce the data and infer magnetic field properties. However, it should be recognised that the inversion process is a very difficult problem and that the currently developed techniques are the best at the moment. Possible errors are related to the use of a finite-sized database for fitting the observed data, along with the assumption that each observed profile corresponds to a unique magnetic field vector. The latter may cause a problem if a number of small-scale plasma and magnetic field structures with varying field strengths lie along the integrated line of sight. Theoretical studies using the polarised radiative transfer equations similar to that used in the whole prominence fine structure model of Gunár \& Mackay (2015a,b) for $\mathrm{H} \alpha$ should be undertaken to consider this. In this approach, magnetic dips with a realistic plasma loading are used to derive the observed profiles instead of considering the magnetic field as a proxy for visible structures.

We have considered the first direct comparison of the observed magnetic field of a prominence with that deduced from a theoretical model. The results show that the observed magnetic field is best represented when the magnetic field of the prominence is represented by a twisted flux rope and not by a sheared arcade. We therefore conclude that the observed magnetic field configuration of the prominence is that of a flux rope. Future studies should expand on these initial studies to consider a variety of prominences across a range of latitudes, along with a wider range of model parameter space.

Acknowledgements. We would like to thank the team of THEMIS for acquiring the observations. DHM would like to thank the UK STFC, ERC and Leverhulme Trust for financial support. Y.S. is supported by NSFC 11473071, 11790302(11790300) and the one hundred talent program of Chinese Academy of Sciences. DHM, BS and ALA participated in ISSI Team 374, "Solving the prominence paradox", led by Nicolas Labrosse where this collaboration was conceived and initiated. CLIMSO is a suite of solar instruments at Pic du Midi (France) operated conjointly by the Observatoire Midi-Pyrénées and the Observateurs Associés, under partial funding from FIDUCIAL. CLIMSO data is freely available through Observatoire Virtuel du Grand Sud Ouest (OVGSO).

\section{References}

Anzer, U., \& Heinzel, P. 2005, ApJ, 622, 714

Aulanier, G., \& Démoulin, P. 1998, A\&A, 329, 1125

Aulanier, G., \& Démoulin, P. 2003, A\&A, 402, 769

Babcock, H. W., \& Babcock, H. D. 1955, ApJ, 121, 349

Bobra, M. G., van Ballegooijen, A. A., \& DeLuca, E. E. 2008, ApJ, 672, 1209

Bommier, V., \& Leroy, J. L. 1998, in IAU Colloq. 167: New Perspectives on Solar Prominences, eds. D. F. Webb, B. Schmieder, \& D. M. Rust, ASP Conf. Ser., 150,434
Bommier, V., Landi Degl'Innocenti, E., Leroy, J.-L., \& Sahal-Brechot, S. 1994, Sol. Phys., 154, 231

Casini, R., López Ariste, A., Tomczyk, S., \& Lites, B. W. 2003, ApJ, 598, L67

Dudík, J., Aulanier, G., Schmieder, B., Bommier, V., \& Roudier, T. 2008, Sol. Phys., 248, 29

Engvold, O. 2015, in Solar Prominences, eds. J. C. Vial, \& O. Engvold, Astrophys. Space Sci. Lib., 415, 31

Gunár, S., \& Mackay, D. H. 2015a, ApJ, 803, 64

Gunár, S., \& Mackay, D. H. 2015b, ApJ, 812, 93

Gunár, S., \& Mackay, D. H. 2016, A\&A, 592, A60

Gunár, S., Schwartz, P., Dudík, J., et al. 2014, A\&A, 567, A123

Gunár, S., Dudík, J., Aulanier, G., Schmieder, B., \& Heinzel, P. 2018, ApJ, 867, 115

Kippenhahn, R., \& Schlüter, A. 1957, ZAp, 43, 36

Kuperus, M., \& Raadu, M. A. 1974, A\&A, 31, 189

Labrosse, N., Heinzel, P., Vial, J.-C., et al. 2010, Space Sci. Rev., 151, 243

Lemen, J. R., Title, A. M., Akin, D. J., et al. 2012, Sol. Phys., 275, 17

Leroy, J. L. 1977, A\&A, 60, 79

Leroy, J. L. 1989, in Dynamics and Structure of Quiescent Solar Prominences, ed. E. R. Priest, Astrophys. Space Sci. Lib., 150, 77

Levens, P. J., Schmieder, B., Labrosse, N., \& López Ariste, A. 2016a, ApJ, 818, 31

Levens, P. J., Schmieder, B., López Ariste, A., et al. 2016b, ApJ, 826, 164

Lionello, R., Mikić, Z., Linker, J. A., \& Amari, T. 2002, ApJ, 581, 718

Lopez Ariste, A. L. 2014, in Nature of Prominences and their Role in Space Weather, eds. B. Schmieder, J. M. Malherbe, \& S. T. Wu, IAU Symp., 300, 370

López Ariste, A., \& Aulanier, G. 2007, in The Physics of Chromospheric Plasmas, eds. P. Heinzel, I. Dorotovič, \& R. J. Rutten, ASP Conf. Ser., 368, 291

López Ariste, A., \& Casini, R. 2002, ApJ, 575, 529

López Ariste, A., \& Casini, R. 2003, ApJ, 582, L51

Luna, M., Su, Y., Schmieder, B., Chandra, R., \& Kucera, T. A. 2017, ApJ, 850, 143

Mackay, D. H., \& van Ballegooijen, A. A. 2009, Sol. Phys., 260, 321

Mackay, D. H., Karpen, J. T., Ballester, J. L., Schmieder, B., \& Aulanier, G. 2010, Space Sci. Rev., 151, 333

Mackay, D. H., Yeates, A. R., \& Bocquet, F.-X. 2016, ApJ, 825, 131

Ouyang, Y., Zhou, Y. H., Chen, P. F., \& Fang, C. 2017, ApJ, 835, 94

Paletou, F., \& Aulanier, G. 2003, in Solar Polarization, eds. J. Trujillo-Bueno, \& J. Sanchez Almeida, ASP Conf. Ser., 307, 458

Sahal-Brechot, S., Bommier, V., \& Leroy, J. L. 1977, A\&A, 59, 223

Schmieder, B., Lin, Y., Heinzel, P., \& Schwartz, P. 2004, Sol. Phys., 221, 297

Schmieder, B., Kucera, T. A., Knizhnik, K., et al. 2013, ApJ, 777, 108

Schmieder, B., Tian, H., Kucera, T., et al. 2014, A\&A, 569, A85

Schmieder, B., López Ariste, A., Levens, P., Labrosse, N., \& Dalmasse, K. 2015, in IAU Symp., eds. K. N. Nagendra, S. Bagnulo, R. Centeno, \& M. Jesús Martínez González, 305, 275

Snodgrass, H. B. 1983, ApJ, 270, 288

Su, Y., \& van Ballegooijen, A. 2012, ApJ, 757, 168

Su, Y., van Ballegooijen, A., Schmieder, B., et al. 2009, ApJ, 704, 341

Su, Y., Gömöry, P., Veronig, A., et al. 2014, ApJ, 785, L2

Su, Y., van Ballegooijen, A., McCauley, P., et al. 2015, ApJ, 807, 144

Su, Y., Liu, R., Li, S., et al. 2018, ApJ, 855, 77

Tandberg-Hanssen, E. 1995, in The Nature of Solar Prominences (Dordrecht: Kluwer Academic Publishers), Astrophys. Space Sci. Lib., 199

van Ballegooijen, A. A. 2004, ApJ, 612, 519 